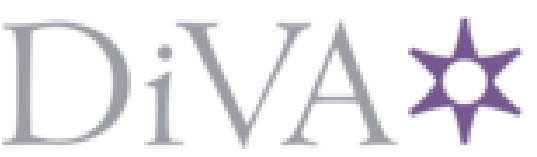

http://www.diva-portal.org

[This is not an article, chapter, of conference paper!] 


\title{
Experimental Evaluation of a Rare Earth-Free Permanent Magnet Generator
}

\author{
Petter Eklund, Jonathan Sjölund, Marcus Berg, Mats Leijon, Member, IEEE and Sandra Eriksson
}

\begin{abstract}
Low speed, high torque machines are used in wind turbines where the turbine rotor is directly connected to the generator. A permanent magnet synchronous generator using high-energy rare-earth permanent magnets (PMs) is one common choice for this application, but rare-earth PMs have supply insecurities and cost risks. A rare-earth free 32-pole PM generator, using ferrite PMs in a spoke-type rotor, for use in a $12 \mathrm{~kW}$ experimental wind turbine is built and tested. Voltages and currents at load and no load are measured, as well as the magnetic field in the end regions of the machine. The generator can deliver the required power at nominal speed and has low harmonic content in the output. The electrical measurements are compared to two-dimensional finite element design calculations and the experimental results are lower than expected from simulations. The measured voltage is lower than expected, requiring a higher current than calculated for the rated power. Three-dimensional magnetic field simulations show that there are leakage flux paths in the end-regions that the two-dimensional design calculations overlook, explaining the discrepancy between simulations and measurements.
\end{abstract}

Index Terms-Permanent magnet generators, Performance evaluation, Spoke-type rotor, Ferrite permanent magnets, End effects, Finite element methods, Experimental evaluation

\section{INTRODUCTION}

$\mathbf{I}$ $\mathrm{N}$ wind power there is a need for low speed, high torque generators when using a gearless drivetrain, where the turbine rotor drives the generator directly. Permanent magnet (PM) excited machines are a common choice as the absence of field coils removes the associated power losses and simplifies construction of the rotor. This also allows for a smaller pole pitch, which is beneficial in low-speed machines, where a large number of poles is needed to avoid long coil ends [1]. The use of direct drive in wind power is desirable, since gearbox failures are a major cause of down-time in wind turbines with a geared drive-train [2]. A gearbox also causes power losses [3].

A popular design is the surface mounted PM rotor which can be easily realized mechanically [4], [5]. A drawback of this kind of rotor is that it works best with high energy PMs, such as those based on samarium-cobalt or neodymiumiron-boron PM materials. The rare-earth elements needed to

The Åforsk Foundation (grant number 12-295) and Carl Trygger Foundation (grant number 15:152) are acknowledged for contributions to the experimental setup. This work was conducted within the STandUP for Energy strategic research framework.

P. Eklund, J. Sjölund, M. Berg, M. Leijon, and S. Eriksson are with the Division of Electricity at Uppsala University, Uppsala, Sweden. (e-mail: see http://www.teknik.uu.se/electricity/contact/ )

M. Leijon is also with the Division of Electrical Machines and Power Electronics, Chalmers University of Technology, Gothenburg, Sweden. (email: matslei@chalmers.se)

Manuscript received October 11, 2018; revised INSERT DATE manufacture these PM materials are either expensive or are associated with supply insecurities and unstable prices. One option is to use ferrite PMs. Ferrite PMs have lower magnetic energy but are cheaper and use more abundant raw materials [6]. The lower remanence of ferrites can be overcome by using a flux concentrating rotor topology with tangentially magnetized magnets placed between magnetically soft poles, such as the spoke-type rotor [7], [8], [9], [10], [11]. This is in contrast with the Halbach array rotor, as in [12], where the soft magnetic poles are replaced by radially magnetized PMs. The spoke-type rotor concept is sometimes also called interior circumferential [13], or tangential interior [14] rotor.

The electrical machine experimentally evaluated in this paper is a 32-pole generator prototype designed for wind power [11]. A prototype has been built and an investigation on the impact of manufacturing tolerances, both mechanical and magnetic, on the air gap magnetic flux density has been made [15] as well as measurements and simulations of the magnetic flux density around its axial ends [16]. A more in-depth investigation of the machine based on electrical measurements and the impact of the magnetic end effects on the electrical output, are the topic of this paper.

Previous work on electrical machines with ferrite magnets are mainly focused on motors [7], [9], [10], [14], [17] or generators [18] with 10 poles or less. Only a few examples of machines with many poles and ferrite magnets have been presented. One example was the Windformer, presented by $\mathrm{ABB}$ in 2000 [19], where a prototype 32-pole generator was built and tested but experimental results were not published. Some other design studies on generators with many poles and ferrite magnets have been presented [6], [20], [21] but the only experimental results found from a ferrite spoke-type rotating generator with many poles were measurement for a 26-pole modular machine in [20]. The study presented a no-load voltage of $94 \%$ of that calculated from magnetic equivalent circuits and also presented some load measurements.

There are several important differences between machines with a large number of poles (such as the 32-pole machine considered here) and machines with fewer poles (10 or less). In a machine with fewer poles, the poles are placed quite close to the shaft, usually made of magnetic material and thereby enabling another leakage path for the magnetic flux. In addition, the smaller the diameter, the closer the poles are placed to each other. In a machine with many poles, the poles are placed on a ring with a rather large circumference, giving smaller angles, both of magnetic field and of the poles, i.e. a longer distance between magnets. However, the length to diameter ratio is usually smaller if the pole-number is high, 
giving possible end effects a larger impact on the output. There is also a difference regarding saliency. Large diameter generators with many poles usually have a small difference between d- and q- axis inductance [20], whereas the motors in [17] for instance have rather high saliency. Finally, a motor is usually operated closer to saturation than a wind power generator at high load.

For spoke-type rotors the induced voltage is often shown to be overestimated by two dimensional (2D) finite element (FE) simulations in publications comparing 2D FE simulations to experiments. In [7] and [9], the experimental EMF is around $8 \%$ lower than the voltages from FE, while in [10] the discrepancy is even larger. None of these papers discusses the discrepancies or possible causes in detail. To find the cause of the discrepancy it is of interest to investigate the three dimensional (3D) field in the machine, especially in the end regions. This is done with respect to the end leakage flux in [22], [17], and to calculate losses in various structural parts in the end regions of the stator in [23]. In [22], 3D simulations and experiments are performed to investigate the leakage flux around the generator shaft, i.e. on the inside of the rotor. In [17], the measured no-load voltage of a spoke-type 8pole motor only differed from simulations with a few percent $(2.6 \%)$, whereas the difference increased significantly at heavy loading due to magnetic saturation. 2D and 3D simulations of the leakage flux at different loading and saturation conditions are presented but measurements of the magnetic flux density was not performed.

In [18], an overhanging rotor for axial flux concentration is presented, where static 3D FE analysis is used to modify a 2D simulation to account for three-dimensional effects such as axial flux concentration. In addition, the air gap magnetic flux density of a non-overhanging rotor was shown to be lower when simulated in $3 \mathrm{D}$ than in $2 \mathrm{D}$. To simplify design work, a thumb role on how to compensate for the $3 \mathrm{D}$ end leakage flux in a 2D simulation would give more accuracy and severely decrease computation time. A first attempt to find an expression for a coefficient for calibrating 2D simulations to 3D flux was presented, based on the 32-pole generator in this study [16]. In [24], a study on magnetic leakage flux on a 10 -pole motor was presented with the focus on proposing a calibration coefficient between 3D and 2D flux.

This paper begins with a presentation of the 32-pole generator and the research methodology and continues with experimental evaluation of electrical parameters and comparison to $2 \mathrm{D}$ simulations. In order to account for the discrepancies found, end effects are investigated with $2 \mathrm{D}$ and $3 \mathrm{D}$ simulations as well as measurements of the magnetic flux density in and outside the air gap.

\section{Fundamental GENERATOR THEORY}

The purpose of a generator is to convert mechanical power into electrical power. To do this a voltage needs to be induced. Faraday's law states that the circulation of the electric field, i.e. the EMF, around a closed path $\partial S$, is equal to the rate of change of magnetic flux through the surface $S$, bounded by
TABLE I

PROPERTIES OF THE INVESTIGATED GENERATOR

\begin{tabular}{lc} 
Quantity & Value \\
\hline Rated power & $12 \mathrm{~kW}$ \\
Nominal speed & $127 \mathrm{rpm}$ \\
Number of poles & 32 \\
Nominal electrical frequency & $33.9 \mathrm{~Hz}$ \\
Number of slots per pole and phase & $\frac{5}{4}$ \\
Maximum diameter of frame & $1.1 \mathrm{~m}$ \\
Overall length (excluding shaft) & $0.48 \mathrm{~m}$ \\
Stator outer diameter & $886 \mathrm{~mm}$ \\
Stator inner diameter & $760 \mathrm{~mm}$ \\
Stator stack length & $224 \mathrm{~mm}$ \\
Air gap length & $6.6 \mathrm{~mm}$ \\
Cross section of cable conductor & $16 \mathrm{~mm}$
\end{tabular}

that path. If $S$ is given a unit normal vector such that $\partial S$ is right-hand oriented, this can be stated as

$$
\mathcal{E}=\oint_{\partial S} \boldsymbol{E} \cdot \mathrm{d} \boldsymbol{l}=-\frac{\mathrm{d}}{\mathrm{d} t} \iint_{S} \boldsymbol{B} \cdot \mathrm{d} \boldsymbol{S}
$$

where $\mathcal{E}$ is the EMF, $\boldsymbol{E}$ the electric field, $\mathrm{d} \boldsymbol{l}$ a directed path segment of $\partial S, t$ is time, $\boldsymbol{B}$ the magnetic flux density, and $\mathrm{d} \boldsymbol{S}$ a directed surface segment.

In a synchronous machine, $\partial S$ is taken to follow one phase of the stationary armature windings. Under some approximations stated below, the RMS value of the EMF of harmonic order $h$, in a winding can be calculated as

$$
\mathcal{E}_{\mathrm{RMS}, h}=\sqrt{8} \pi D l \hat{B}_{\rho, h} f N_{\mathrm{eff}}
$$

where $D$ is the stator inner diameter, $l$ the axial length of the stator, $\hat{B}_{\rho, h}$ the amplitude of the $h$ th order harmonic of the radial magnetic flux density on the stator inner surface, and $f$ the electrical frequency. The factor $N_{\text {eff }}$ is the effective number of turns per pole, i.e. the number of physical turns per pole, multiplied by correction factors to account for phase shifts between turns, the coil pitch, and if the stator or rotor is skewed. The approximations used in the derivation of (2) are three. The first is that $\boldsymbol{B} \cdot \mathrm{d} \boldsymbol{S}=0$ outside the machine. The second is that there is no axial variation of $\boldsymbol{B}$. The third is that each slot and the winding turns contained therein are small enough to be considered an axial line on the inner periphery of the stator.

\section{THE GENERATOR}

The investigated generator is designed for a $12 \mathrm{~kW}$ experimental wind turbine, and built according to the design described in [11]. The generator is a result of a study on substituting a neodymium-iron-boron surface mounted rotor with a ferrite PM rotor for the same stator [5], [6], [11]. A design requirement for the generator was to have a low rated load angle, in order to have a large overload torque capability to enable electric control of the wind turbine [5]. The generator was designed with a fractional slot winding to decrease cogging, which is of importance for wind turbine start-up. Some characteristics are listed in Table I. Fig. 1 shows a photograph of the generator in the test frame and a closeup of the air gap. The generator has a self-supporting design and does not conform with any standard electric 


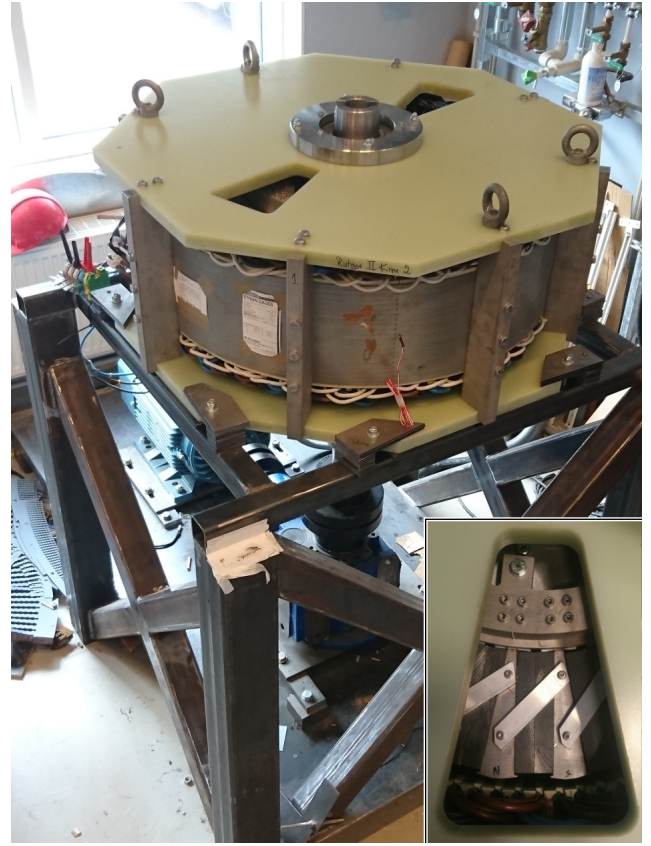

Fig. 1. The investigated generator on the test frame and a close-up of the air gap, showing rectangular ferrite PMs placed between steel pole pieces. The octagonal end board is $1 \mathrm{~m}$ from side to side.

machine frame size. The mechanical design is elaborated in [11]. The rotor of the generator is of spoke-type design and employs ferrite PMs. The ordered ferrite magnets were supposed to be of grade Y40. However, when measured they showed to have lower remanence than expected [11]. The ferrite generator is compared to the previously built rare-earth generator [11], [25]. The ferrite generator performs well in comparison regarding protection against demagnetization [25]. The ferrite generator was designed with slightly lower voltage than the rare-earth generator, partly since the experimental machines were built with rather large airgap. However, both machines perform well at rated power [11].

The rotor design is chosen to fit as much PM material as possible for a given stator diameter, since the stator is kept the same. As ferrites are relatively cheap, some excessive PM material is considered managable to compensate for the leakage flux inherently caused by a spoke-type configuration. In [25], the risk for irreversible demagnetization under shortcircuit events is studied for this generator at both low and high temperatures. The results show very small areas of irreversible demagnetization in the corners of the magnets. The rotor support structure, connecting the poles to the shaft, is made of non-magnetic aluminum to decrease leakage flux. The shaft is made of magnetic steel. The stator is wound with lowvoltage cables, and has slots with grooves to help hold the cables in place. The stator has clamping boards designed to compress both stator yoke and teeth. Despite this, the teeth are not sufficiently compressed axially to prevent the laminations from fanning out and the stator appears to be $0.5 \mathrm{~mm}$ to $2 \mathrm{~mm}$ longer on the inner periphery than on the outer periphery. The generator is mounted in a test frame with a driving induction motor connected to the generator by a right-angle gearbox placed below the generator. The induction motor is rated at $30 \mathrm{~kW}$ and $1470 \mathrm{rpm}$ and is inverter-fed in order to facilitate variable speed operation. The gearbox has a speed ratio of 8:1.

Before inserting the magnets into the rotor, the magnetization was indirectly measured for each magnet, by measuring the magnetic flux density on the surface of the magnet with a Gauss/Tesla meter, with no other magnetic material in the vicinity (see Section IV-B). The magnetic flux density measured was lower than expected for a PM block of that size and magnetization grade. A 3D simulation was performed on a magnet placed inside a large volume of air, and the remanence of the magnet was tuned to fit the measurement, giving an average remanence estimated to $0.38 \mathrm{~T}$ instead of the nominal $0.45 \mathrm{~T}$. The air gap length in front of the pole was measured to $6.6 \mathrm{~mm}$ in average for all poles, while the design value was $7 \mathrm{~mm}$.

\section{Measurements and Instrumentation}

The quantities measured on the machine are: voltage at no load at different speeds; voltage, current and power at full load and multiple part load cases; and stator winding resistance. The magnetic flux density in the air gap and in the end region are also measured.

\section{A. Electrical Measurements}

The no load terminal voltage is measured at increasing speeds in ten increments from standstill up to nominal speed. The slow ramping of the speed is done both to check the voltage along the way, but also to verify the mechanical integrity of the generator and the attachment to the test frame. The lineto-neutral voltage is measured during the speed ramping. At full speed also the line-to-line voltage is measured.

For the load tests, two of the line-to-line voltages and two of the currents are measured; this allows the power to be computed using the two-watt-meter method as the neutral of the star connected winding is not connected to the load. The load resistance is adjusted to achieve the rated load of $12 \mathrm{~kW}$ with balance between the phases. Line-to-neutral voltages in the load case are calculated from line-to-line voltages assuming that the zero sequence voltage is zero; this is equivalent to using an artificial neutral created by star connection of three identical impedances. For the no-load test three differential voltage probes are used to measure all three line-to-neutral voltages directly, using the neutral of the star connected generator winding.

In the no-load and load tests, data are logged using a PC oscilloscope together with differential voltage probes, with an attenuation of $1 / 100$, and current probes, with a gain of $10 \mathrm{mV} / \mathrm{A}$. The PC oscilloscope used is a Pico Technology PicoScope 3425. The accuracy of the oscilloscope is specified as $\pm 1 \%$ of range, resulting in $\pm 2 \mathrm{~V}$ for the no-load tests, and $\pm 5 \mathrm{~V}$ or $\pm 1 \mathrm{~A}$ for the load tests. The differential voltage probes used are two Pico Technology TA041 and one Testec TT-SI 9001 (only for the no-load voltage). For all the differential voltage probes the accuracy is specified as $\pm 14 \mathrm{~V}$ in the used configuration. The current probes used are Agilent 1146A AC/DC Oscilloscope Current Probes. The specified accuracy 
in the used $10 \mathrm{mV} / \mathrm{A}$ mode is $\pm(4 \%$ of reading $+50 \mathrm{~mA})$ for currents below $40 \mathrm{~A}$ and $\pm(15 \%$ of reading $+100 \mathrm{~mA})$ for larger currents.

Cross calibration of the voltage measurements against a Rigol DM3058E Digital Multimeter (DMM) indicates that the maximum error of an individual sample in the voltage measurements is less than $\pm 3.98 \mathrm{~V}$ in the load cases and $\pm 1.36 \mathrm{~V}$ in the no-load case. Cross calibration of the current measurements using the same DMM as the voltage calibration indicates that the maximum error of an individual sample is less than $\pm 1.54 \mathrm{~A}$ (including the accuracy of the DMM) inside the range of $-58 \mathrm{~A}$ to $58 \mathrm{~A}$ for DC. For sinusoidal $\mathrm{AC}$ at $50 \mathrm{~Hz}$ the maximum error is less than $\pm 0.54 \mathrm{~A}$ for the RMS current, tested for $47 \mathrm{~A}$. The DMM is limited to $10 \mathrm{~A}$. To test for currents above $10 \mathrm{~A}$, a current close to the upper limit is used and the conductor is passed multiple times through the gap of the current probe. A mainly inductive load is used to reduce harmonics in the test current.

Resistance of the stator winding is measured using a Metra Hit 17 milliohmmeter and a four wire measurement connection. The specified accuracy of the instrument in the used range is $\pm\left(0.1 \%\right.$ of reading $\left.+2 \times 10^{-4} \Omega\right)$. Measurements are made from each phase terminal to the neutral terminal. The neutral terminal is used instead of the actual neutral point to avoid removing the insulation on the neutral point. This will make the measured phase resistance slightly larger than the true value since an additional $0.6 \mathrm{~m}$ of $16 \mathrm{~mm}^{2}$ copper cable (corresponding to $0.6 \mathrm{~m} \Omega$ ) and two extra contact points are included in the circuit.

\section{B. Magnetic Flux Density Measurements}

Measurements of the radial magnetic flux density component are carried out along an axial line segment. This line segment has one end in the air gap, where a pole shoe faces a stator tooth, and the other end some distance outside the machine. The location of the line segment is indicated in Fig. 2. The pole with the highest magnetic flux density is chosen. The measured magnetic flux density distribution is compared with simulated distributions. Details of the simulations are given in Section V-B.

An FW Bell 5180 Gauss/Tesla meter is used to measure the magnetic flux density. The specified accuracy for values over $30 \mathrm{mT}$ is $\pm(0.6 \%+3$ counts $)$, where one count is $0.1 \mathrm{mT}$ below $300 \mathrm{mT}$ and $1 \mathrm{mT}$ for larger values. Below $30 \mathrm{mT}$ specified accuracy is $\pm(0.8 \%+0.04 \mathrm{mT})$. The percentages are of the measured value. The measurements are done manually in axial steps of $2 \mathrm{~mm}$ starting $40 \mathrm{~mm}$ outside of the machine, and ending $52 \mathrm{~mm}$ into the air gap. The systematic error is estimated to be up to $\pm 2 \mathrm{~mm}$ in absolute axial position, but the relative axial positions should be accurate to $\pm 0.25 \mathrm{~mm}$. The error in absolute position in the radial-tangential plane is more difficult to estimate and is probably higher. The relative position in the plane should also be less accurate but the error is not as prominent as the error in absolute position.

\section{Propagation of Errors}

In order to evaluate the measurement error for the different quantities, the propagation of errors needs to be considered.
The propagation of errors is calculated according to the method presented in [26] but adapted for the measurements performed here. Maximum error is used, as calculation of average error relies on the assumption that the errors are not correlated, which is not likely to be true for multiple samples using the same instrument. Let $\Delta x_{i},(i=1,2, \ldots n)$ be the maximum error of the measured quantities $x_{i}$. The maximum error for an RMS value of a signal with $N$ samples is

$$
\Delta X_{\mathrm{RMS}}=\sum_{i=1}^{N}\left|x_{i} \Delta x_{i}\right|\left(N \sum_{j=1}^{N} x_{j}^{2}\right)^{-1 / 2}
$$

The average power is computed from simultaneously sampled values of two line-to-line voltages, $V_{a b, i}, V_{b c, i}$, and currents in two of the phases, $I_{a, i}, I_{c, i}$, using the two-watt-meter method. The subscripts $a, b, c$ denote the different phases, while subscript $i$ is the index of the sample. The power in sample $i$ is then computed as

$$
P_{i}=V_{a b, i} I_{a, i}-V_{b c, i} I_{c, i}
$$

The error for the mean power is

$$
\begin{array}{r}
\Delta P=\frac{1}{N} \sum_{i=1}^{N}\left(\left|\Delta V_{a b, i} I_{a, i}\right|+\left|V_{a b, i} \Delta I_{a, i}\right|+\ldots\right. \\
\left.\ldots\left|\Delta V_{b c, i} I_{c, i}\right|+\left|V_{b c, i} \Delta I_{c, i}\right|\right)
\end{array}
$$

\section{Simulations}

There are two sets of electromagnetic simulations made. The first of these are the repeated design simulations, described in [11], but with air gap length and PM remanence as reported in [15]. The second of these are three-dimensional and twodimensional simulations made to investigate the impact of end effects. Both models are implemented using the Rotating Magnetic Machinery module of COMSOL Multiphysics®.

\section{A. Design Simulations}

The design calculations use finite elements to solve for the magnetic field in a 2D cross section of the geometry, shown in Fig. 2. Materials are modeled using a relative permeability for the air and windings, relative recoil permeability and remanent flux density for the PMs, and a saturating BH-curve for the steel. The magnetic field is assumed to be only in the plane of the cross section and all currents are assumed to be perpendicular to the plane. Under this assumption only the outof-plane component of the magnetic vector potential is needed. The current distribution in the winding turns is assumed to be homogeneous, i.e. neglecting skin effect and proximity effect. Eddy currents in other materials are also omitted from the model by setting conductivity to zero everywhere. Since the machine geometry is repeated every $45^{\circ}$, only one repeating sector is simulated with periodic boundary conditions (BCs) in order to reduce the computational effort. The mesh is fine enough to ensure that any numerical error is negligible; this means that the errors in the simulations are modeling errors.

From (2) the most important parameters for the voltage can be identified. In the simulations the stator bore is a mathematical cylinder with a well-defined diameter and length. In reality 


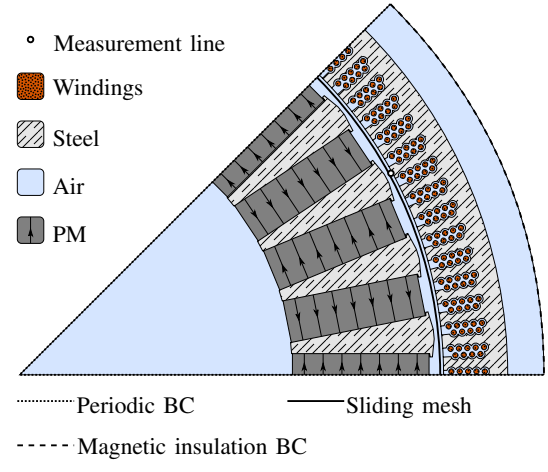

Fig. 2. The 2D simulation geometry. Boundary conditions (BCs) and materials are indicated. The arrows on the hatching of the PMs show the direction of magnetization. The ring in front of the second pole indicates the location in the plane of an axial line segment where the radial component of the magnetic flux density is measured. The line segment starts inside the air gap and extends outside the end of the machine.

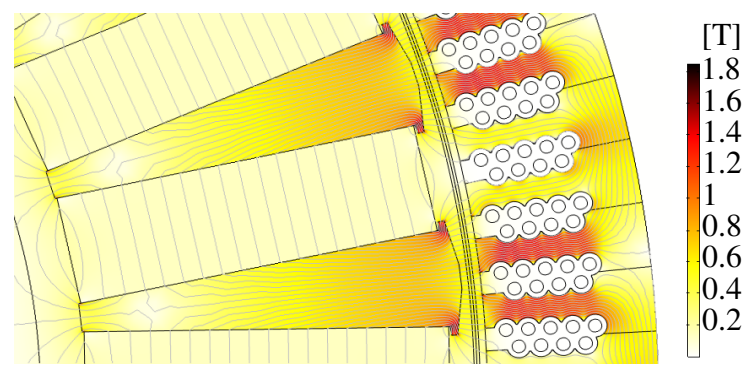

Fig. 3. The magnetic flux density and field lines at no-load from the 2D FE design simulations for part of the geometry.

the diameter measured will depend both on angle and axial position for which it is taken due to manufacturing tolerances. Similarly, the length of a real stator will be ambiguous, due to manufacturing tolerances such as uneven compression of the stack of laminations, or burrs on the laminations. For the 2D FE simulations, the machine length is of extra importance, as many quantities are computed per unit length and then multiplied by the machine length. Much of the uncertainties in both the cross section geometry and the material properties goes into $\hat{B}_{\rho, h}$. Some of the uncertainties in the cross section geometry also goes into $N_{\text {eff }}$. The number of physical turns of the winding should be accurate and the impact of the uncertainty in the position of the slot should not have any great impact on the winding factors. The electrical frequency is unambiguous to measure and accurate instrumentation is readily available. It is, however, not possible to keep the frequency exactly constant under experimental conditions. Some uncertainty, depending on the inertia of the system and the speed controller, will therefore be present. Overall, the 2D simulations should be reasonably accurate in predicting the magnetic flux density inside the machine, at sufficient distance from the machine ends. The magnetic flux density from the design calculations at no-load is shown in Fig. 3.

\section{B. Simulations to Investigate End Effects}

A spoke-type rotor has a large end surface on each of the pole pieces. These end surfaces are close to being mag- netic isopotentials separated by the length of a PM (along magnetization) and the magnetic scalar potential takes its extreme values on either side of the PMs. This presents a path for leakage flux, possibly of low reluctance, that is entirely neglected in the 2D simulation. Therefore, 3D simulations are needed to accurately simulate the end effects. 3D simulations of the end leakage flux was previously presented for this generator in [16], showing that most leakage flux occurs above the rotor and does not enter the stator. The simulation methodology has been improved in several ways since then: the mesh is more detailed in and above the air gap, an error in the computation of the radial component of the flux density has been corrected, and the axial lengths for the stator, pole shoes and magnets have been modified to better resemble the prototype.

The simulations to investigate the end effects are made only for a stationary no-load case. The magnetic scalar potential formulation of magnetostatics is used, in order to reduce computational complexity in the $3 \mathrm{D}$ case, as there are no currents, and also in the 2D case for consistency. The windings are removed from the geometry and the shape of the slots is simplified in order to make meshing less complex. The rest of the cross section geometry is the same as in Fig. 2. The 2D geometry is extruded in the axial direction, and a cylinder of free space around the extruded geometry is added to create the 3D geometry. The distance of free space between the machine geometry and the outer boundary of the simulation volume is at least half of the outer radius of the stator.

In the 3D simulations, mirror symmetry is used in the axial direction by setting the normal component of the magnetic flux density on the axial symmetry plane to zero; half of the computational domain can then be removed. Periodic BCs are applied in the same manner as in the design simulations. On the remaining exterior boundaries the normal component of the magnetic flux density is also set to zero. The mesh is made extra fine along the axial line, located as indicated in Fig. 2, that corresponds to the location of the measured radial magnetic flux density, in order to improve the accuracy of the computed magnetic flux density profile. The remanent magnetic flux density of the PM material is tuned to make the magnitude of the simulated magnetic flux density match that of the measurements inside the generator based on the selected pole.

\section{RESULTS AND DISCUSSION}

\section{A. Generator Performance}

A summary of the measurements and design simulations is made in Table II. In the 2D FE design simulations used for Table II and Fig. 4, the air gap length is set to $6.6 \mathrm{~mm}$ and the PM remanence is set to $0.38 \mathrm{~T}$ to match the mean values measured in the prototype [15].

The no-load voltage is measured at ten different speeds up to nominal speed. The no-load voltage is found to be proportional to the speed with a constant of $0.93 \pm 0.03 \mathrm{~V} / \mathrm{rpm}$. In the simulations the value is $0.998 \mathrm{~V} / \mathrm{rpm}$. The maximum deviation between the fit and measurement is $0.35 \%$ of the value. The harmonic contents of the experimental and simulated no-load 
TABLE II

COMPARISON OF MEASUREMENTS AND 2D FE SIMULATIONS. CURRENTS AND VOLTAGES ARE GIVEN AS RMS VALUES. VOLTAGES ARE LINE-TO-NEUTRAL. THE ERROR COLUMN GIVES MAXIMUM \pm ERRORS OF THE MEASURED VALUES.

\begin{tabular}{|c|c|c|c|c|c|}
\hline Quantity & \multicolumn{3}{|c|}{ Experimental } & \multirow{2}{*}{$\frac{\operatorname{Error}( \pm)}{0.79}$} & Simulated \\
\hline Average power $[\mathrm{kW}]$ & \multicolumn{3}{|c|}{12.02} & & 12.06 \\
\hline Phase & a & $\mathrm{b}$ & $\mathrm{c}$ & & \\
\hline No load voltage [V] & $\overline{118.2}$ & 117.5 & $\overline{118.1}$ & 1.2 & 126.7 \\
\hline Full load voltage $[\mathrm{V}]$ & 110.4 & 110.3 & 110.4 & 3.6 & 120.0 \\
\hline Full load current $[\mathrm{A}]$ & 36.4 & 36.3 & 36.3 & 1.5 & 33.36 \\
\hline Resistance $[\mathrm{m} \Omega]$ & 141 & 154 & 151 & 0.4 & 150.0 \\
\hline Ohmic losses per phase [W] & 186 & 203 & 199 & 17 & 167 \\
\hline Synchronous inductance $[\mathrm{mH}$ & & 3.1 & & & 3.03 \\
\hline
\end{tabular}

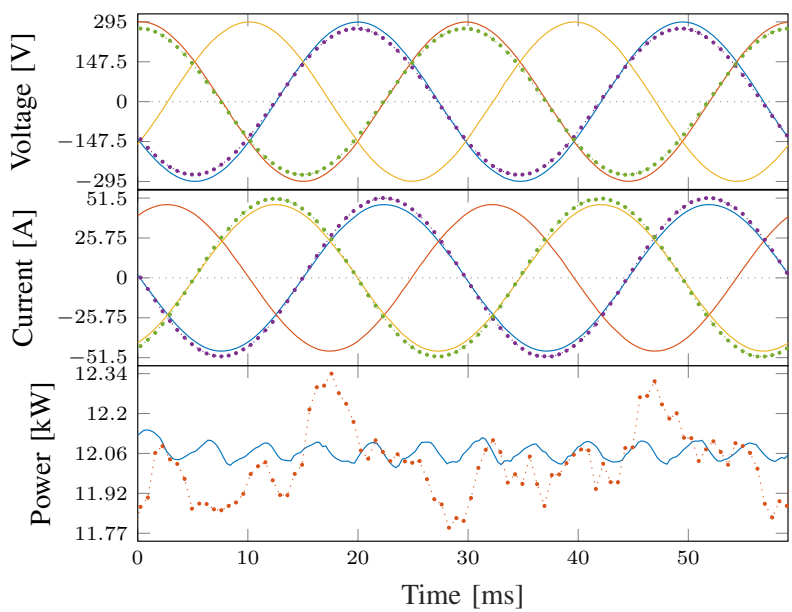

Fig. 4. The line-to-line terminal voltage, terminal currents and power at full load. Solid lines are from 2D FE simulations, while dots connected by dotted lines are from measurements. The load resistances have been tuned to reach an average power of $12 \mathrm{~kW}$.

voltages are shown in Fig. 5; flat-top windowing is used on the experimental data in order to get accurate amplitude information. The difference between phases is small and therefore only one phase is shown. There are some discrepancies between experiments and simulations, but the overall harmonic content is low. The largest harmonic is the third, which is about two orders of magnitude smaller than the fundamental component. There are also some half order harmonic components. Half order harmonics are expected with $\frac{5}{4}$ slots per pole and phase, as the stator winding is repeated only every two pole pairs. The slot harmonics can be seen at $6 \frac{1}{2}$ of the fundamental frequency. The half and three halves components are more pronounced in the experiments than in the simulations, probably due to manufacturing tolerances. Differences, possibly with the same origin or due to measurement errors, are also apparent for harmonics of order $6 \frac{1}{2}$ and 7 .

The voltage drop as a function of current, for both simulation and experiment, is shown in Fig. 6. These results confirm that no significant saturation is present in the normal operating region, as expected. Assuming a round rotor, the synchronous inductance can be estimated from the voltage drop at full load to $3.1 \mathrm{mH}$ in the experimental case and to $3.03 \mathrm{mH}$ in the simulations. The simulations do not include any modeling of the end winding inductance. From simulations the ratio of the

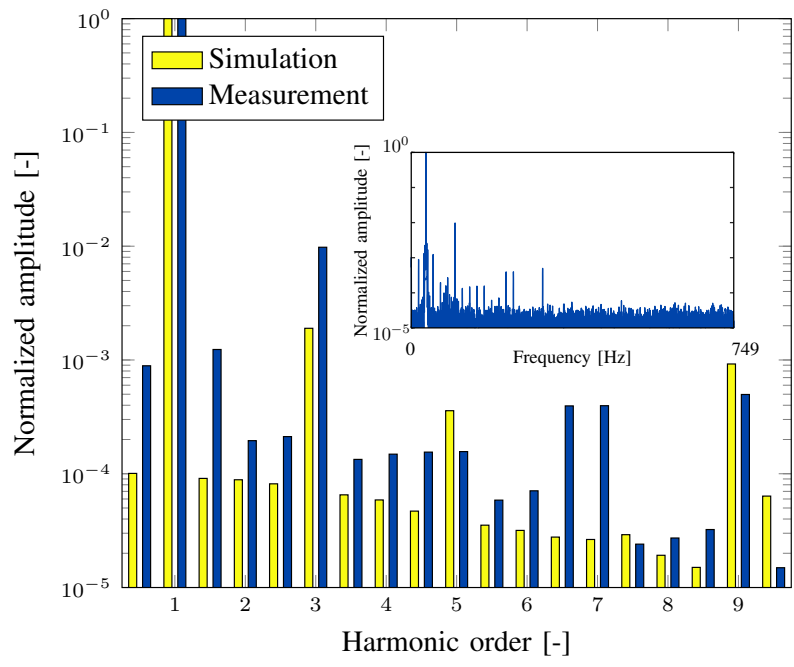

Fig. 5. Harmonic content of measured and simulated no-load voltage presented as normalized amplitude. The inset shows the complete amplitude spectrum of the measured data, from zero up to the Nyquist frequency. The simulated voltage are from 2D FE simulations.

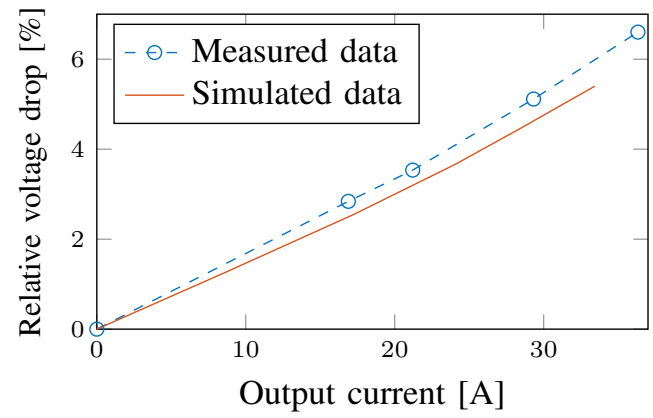

Fig. 6. Relative voltage drop over current. No-load line-to-line voltage is $205 \mathrm{~V}$ in the experiment and $220 \mathrm{~V}$ in the 2D FE simulation. Maximum current corresponds to $12 \mathrm{~kW}$ in both cases.

direct axis and quadrature axis inductances is estimated to be either 0.976 or 1.07 , depending on calculation method. The first value is obtained by finding the slope of a fit of flux linkage over current, computed using static FE. The second value is computed from the voltage drop at load. As the rotor is very close to non-salient the round rotor approximation should be appropriate; it is therefore not expected to be feasible to measure the saliency.

The line-to-line terminal voltage, terminal currents, and output power in a full load test with resistive load are shown in Fig. 4. Both experimental and simulation data are shown. It appears that there are some differences between the phases. This is to be expected, as in any real machine there will be some asymmetries due to manufacturing tolerances. This is mainly visible in the output power, which in the simulations is close to being periodic with a frequency of six times the electrical frequency, whereas in the experiment it has some peaks that are larger once per electrical period. This is consistent with the results from Table II, showing differences in induced voltages and currents between the phases. 


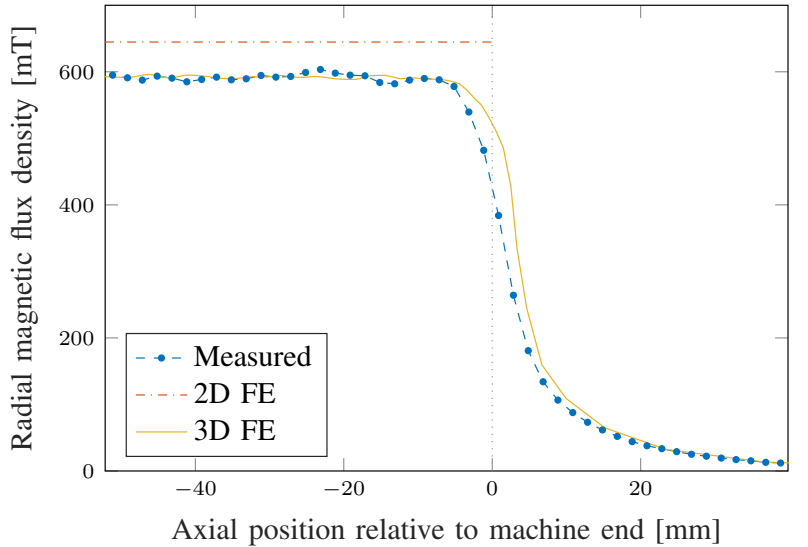

Fig. 7. Radial component of the magnetic field on an axial line in the air gap and end region. Measurements are made for one pole selected for its high field. The simulated remanence is adjusted so the magnetic flux density from the $3 \mathrm{D}$ simulations match the experiments. The field of a $2 \mathrm{D}$ simulation is included for comparison.

\section{B. End Effects}

Fig. 7 shows the radial component of the magnetic flux density along the axial line segment located as indicated in Fig. 2, starting in the air gap, and ending some distance outside the machine. The measured axial distribution of the magnetic flux is similar to the distribution from the 3D simulation where the remanence has been adjusted to make the magnitudes match inside the generator for the selected pole. A remanence of $0.41 \mathrm{~T}$ is used here, as this matches the measurements for this high field pole. The comparison between measurement and 3D simulation is done in an interval starting at $-51.1 \mathrm{~mm}$ to $-22.4 \mathrm{~mm}$ : $-51.1 \mathrm{~mm}$ being the innermost measured value of the prototype. The steep drop in magnetic flux when exiting the stator is shifted by a few millimeters between simulations and measurements, but this difference is similar in magnitude to the measurement accuracy of the axial position. Uncertainty of finding the same position in the radial-tangential plane for measurement and simulation, together with a suboptimal alignment between the selected pole shoe and tooth, result in a slightly different magnitude of the radial flux density compared to [15]. Disregarding the slight irregularities of the measured flux density in Fig. 7, the measured flux density converge to a single value inside the machine at a distance of more than $20 \mathrm{~mm}$ from the ends. A second measurement series is made, but there is no noticeable difference between the measurement series. The difference between 3D and 2D FE is in line with previous findings reported in [18], where 3D FE simulations of a non-overhanging spoke rotor gave lower magnetic flux density in the air gap than a 2D FE simulation.

The behavior of the air gap magnetic flux density in response to changes in machine length is simulated and the result is shown in Fig. 8. As the magnetic flux density is converging inside the machine, the 3D distribution is represented by a single value and compared to the $2 \mathrm{D}$ case. Increasing the machine length while keeping the diameter constant increases the amplitude but does not change the shape of the flux density distribution. As the machine becomes more elongated the difference between 2D and 3D decreases asymptotically.

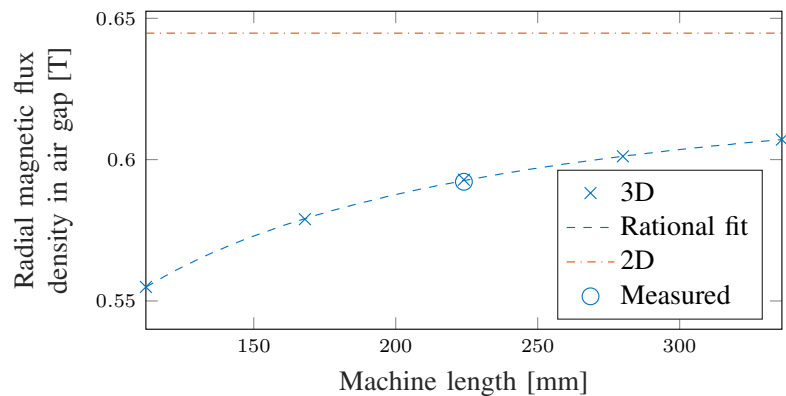

Fig. 8. Magnitude of radial magnetic flux density inside the stator core over machine length from FE simulations. The values given are the spatial mean value for the middle $80 \%$ of the machine. The measured value is the mean of the internal measured values from $-51.1 \mathrm{~mm}$ to $-22.4 \mathrm{~mm}$ in Fig. 7. The rational fit has a first order polynomial as both numerator and denominator.

Fitting a rational function to the data from 3D simulations of different machine lengths, with a first order polynomial as both numerator and denominator, and extrapolating to infinite machine length gives a value with about $1 \%$ difference to the $2 \mathrm{D}$ value. This behavior is consistent with that of a lumped magnetic circuit, where the MMF and reluctance of the PM are connected in series with two parallel-connected reluctances, one representing the in-plane flux paths modeled with 2D FE and the other representing the end leakage paths that only 3D FE captures. The reluctances of the PM and the in-plane flux paths are both inversely proportional to the length of the machine. This observation could form the basis for a general method of correcting the 2D FE to improve the accuracy when simulating machines with spoke-type rotors.

The terminal voltage is about $7 \%$ to $8 \%$ lower in experiments than in the simulations, resulting in a higher current for the same output power. This causes a $17 \%$ increase in ohmic losses compared to simulations. The remanence and air gap length of the simulations have been corrected to match those of the prototype, to better test the accuracy of the simulations. Especially an incorrect remanence of the PMs has large impact on the predicted output voltage; therefore this correction is important. The stacking effects on active length are already accounted for, since the rotor and the stator are of equal lengths, if disregarding fanning out of the stator laminations in the stator teeth. While there is some uncertainty in the measurements of the stator inner diameter and the stator stack length, the influence of these, judging from (2), is not large enough to explain the difference in voltage. This leaves 3D end effects as a possible explanation.

If the voltage from the $2 \mathrm{D}$ simulation is scaled by the ratio between the air gap radial magnetic flux density obtained by $3 \mathrm{D}$, and that obtained for $2 \mathrm{D}$, i.e. $92 \%$, a scaled simulated no load voltage that is slightly lower than the measured voltage is obtained. The difference is nearly small enough to fall within the tolerance of the measurements. If it is not a measurement error it can possibly be explained by the behavior of the radial magnetic flux density near the end of the machine. In Fig. 7, it can be seen that the area bounded by a horizontal line on the level of the near constant magnetic flux density inside the stator, the magnetic flux density curve, and the end of the 
machine (i.e. axial position 0) is smaller than the area bounded by the magnetic flux density curve, zero magnetic flux density and the machine end. This corresponds to that the magnetic flux passing over to the stator outside the machine end is larger than the deficiency in flux near the stator end. While not all of this magnetic flux is passing through the armature winding, either by going into the stator core or by passing through the end windings, some of it most likely does.

\section{CONCLUSIONS}

A unique, 32-pole spoke-type ferrite generator has been experimentally evaluated. Measurements of voltage and current have been performed at load and at no load, and compared to design simulations. The measured voltage has a low harmonic content and the generator can deliver the rated power. It is demonstrated that a spoke-type rotor with ferrite PMs is a viable option for a rare-earth metal free PM synchronous generator.

It is found that the induced voltages are lower than calculated by the $2 \mathrm{D}$ simulation, even when effects of manufacturing tolerances have been taken into account. The discrepancy is believed to originate from magnetic leakage flux at the axial ends. The leakage flux is investigated through 3D simulations and experiments and is found to satisfactorily explain the difference. The quotient between the experimental and simulated no load voltage rms is 0.93 whereas the quotient between the 2D and 3D simulated magnetic flux densitiy is 0.92 . The similar quotients can explain the discrepancy between experiment and 2D simulation.

When changing the machine length in the simulations, the magnetic flux density in the air gap, away from the machine ends, behaves as a fraction of two first order polynomials that asymptotically approaches the value in $2 \mathrm{D}$ for long machines. This behavior can be predicted using a simple magnetic circuit model. A possibility for future work is to use this to devise a method to correct 2D FE simulations of spoke-type rotors to take the end-leakage flux into account.

\section{REFERENCES}

[1] E. Spooner and A. C. Williamson, "Direct coupled, permanent magnet generators for wind turbine applications," IEE Proceedings - Electric Power Applications, vol. 143, no. 1, pp. 1-8, Jan 1996.

[2] J. Ribrant and L. M. Bertling, "Survey of failures in wind power systems with focus on swedish wind power plants during 1997-2005," IEEE Transactions on Energy Conversion, vol. 22, no. 1, pp. 167-173, March 2007.

[3] P. M. Marques, C. M. Fernandes, R. C. Martins, and J. H. Seabra, "Power losses at low speed in a gearbox lubricated with wind turbine gear oils with special focus on churning losses," Tribology International, vol. 62, pp. 186-197, 2013.

[4] Y. Chen, P. Pillay, and A. Khan, "PM wind generator topologies," IEEE Transactions on Industry Applications, vol. 41, no. 6, pp. 1619-1626, Nov 2005.

[5] S. Eriksson, A. Solum, M. Leijon, and H. Bernhoff, "Simulations and experiments on a $12 \mathrm{~kW}$ direct driven PM synchronous generator for wind power," Renewable Energy, vol. 33, no. 4, pp. 674-681, 2008.

[6] S. Eriksson and H. Bernhoff, "Rotor design for PM generators reflecting the unstable neodymium price," in XXth International Conference on Electrical Machines, Marseille, France, Sept 2012, pp. 1419-1423.

[7] M. Kimiabeigi, J. D. Widmer, R. Long, Y. Gao, J. Goss, R. Martin, T. Lisle, J. M. S. Vizan, A. Michaelides, and B. Mecrow, "Highperformance low-cost electric motor for electric vehicles using ferrite magnets," IEEE Transactions on Industrial Electronics, vol. 63, no. 1, pp. 113-122, Jan 2016.
[8] Q. Chen, G. Liu, W. Zhao, L. Sun, M. Shao, and Z. Liu, "Design and comparison of two fault-tolerant interior-permanent-magnet motors," IEEE Transactions on Industrial Electronics, vol. 61, no. 12, pp. 66156623, Dec 2014.

[9] S. J. Galioto, P. B. Reddy, A. M. EL-Refaie, and J. P. Alexander, "Effect of magnet types on performance of high-speed spoke interior-permanentmagnet machines designed for traction applications," IEEE Transactions on Industry Applications, vol. 51, no. 3, pp. 2148-2160, May 2015.

[10] M. R. Mohammad, K. Kim, and J. Hur, "Design and analysis of a spoke type motor with segmented pushing permanent magnet for concentrating air-gap flux density," IEEE Transactions on Magnetics, vol. 49, no. 5, pp. 2397-2400, May 2013.

[11] P. Eklund, S. Sjökvist, S. Eriksson, and M. Leijon, "A complete design of a rare earth metal-free permanent magnet generator," Machines, vol. 2, no. 2, pp. 120-133, 2014. [Online]. Available: http://www.mdpi.com/2075-1702/2/2/120

[12] Y. Shen, G. Y. Liu, Z. P. Xia, and Z. Q. Zhu, "Determination of maximum electromagnetic torque in PM brushless machines having twosegment Halbach array," IEEE Transactions on Industrial Electronics, vol. 61, no. 2, pp. 718-729, Feb 2014

[13] K. T. Chau, C. C. Chan, and C. Liu, "Overview of permanent-magnet brushless drives for electric and hybrid electric vehicles," IEEE Transactions on Industrial Electronics, vol. 55, no. 6, pp. 2246-2257, June 2008.

[14] C. Suo, K. Yang, G. Xiao, W. Tu, and L. Tang, "Optimal design of rotor topology of PM synchronous machine for high speed electric tools," in 2017 20th International Conference on Electrical Machines and Systems (ICEMS), Aug 2017, pp. 1-5.

[15] P. Eklund and S. Eriksson, "Air gap magnetic flux density variations due to manufacturing tolerances in a permanent magnet synchronous generator," in XXII International Conference on Electrical Machines (ICEM), Lausanne, Switzerland, Sept 2016, pp. 93-99.

[16] P. Eklund, J. Sjölund, S. Eriksson, and M. Leijon, "Magnetic end leakage flux in a spoke type rotor permanent magnet synchronous generator," in 19th International Conference on Electrical Machines and Drives, Madrid, Spain, March 2017.

[17] W. Kakihara, M. Takemoto, and S. Ogasawara, "Rotor structure in $50 \mathrm{~kW}$ spoke-type interior permanent magnet synchronous motor with ferrite permanent magnets for automotive applications," in IEEE Energy Conversion Congress and Exposition, Denver, Colorado, U.S.A., Sept 2013, pp. 606-613.

[18] K.-C. Kim and J. Lee, "The dynamic analysis of a spoke-type permanent magnet generator with large overhang," IEEE Transactions on Magnetics, vol. 41, no. 10, pp. 3805-3807, Oct 2005.

[19] M. Dahlgren, H. Frank, M. Leijon, F. Owman, and L. Walfridsson, "Windformer - wind power goes large scale," ", $A B B$ Review, vol. 3 , pp. 31-37, 2000.

[20] E. Spooner, A. C. Williamson, and G. Catto, "Modular design of permanent-magnet generators for wind turbines," IEE Proceedings Electric Power Applications, vol. 143, no. 5, pp. 388-395, 1996.

[21] N. A. Bhuiyan and A. McDonald, "Assessment of the suitability of ferrite magnet excited synchronous generators for offshore wind turbines," in EWEA Offshore 2015, Copenhagen, Denmark, March 2015.

[22] X. Ge, Z. Q. Zhu, J. B. Li, and J. T. Chen, "A spoke-type IPM machine with novel alternate airspace barriers and reduction of unipolar leakage flux by step-staggered rotor," in IEEE International Electric Machines \& Drives Conference, Couer d'Alene, Idaho, U.S.A., May 2015, pp. 53-59.

[23] H. M. Hämäläinen, J. Pyrhönen, J. Nerg, and J. Puranen, "3-D finite element method analysis of additional load losses in the end region of permanent-magnet generators," IEEE Transactions on Magnetics, vol. 48, no. 8, pp. 2352-2357, Aug 2012.

[24] S. G. Lee and W. Kim, "A study on the axial leakage magnetic flux in a spoke type permanent magnet synchronous motor," in IEEE International Electric Machines and Drives Conference (IEMDC), Miami, Florida, May 2017, pp. 1-6.

[25] S. Sjökvist, P. Eklund, and S. Eriksson, "Determining demagnetisation risk for two PM wind power generators with different PM material and identical stators," IET Electric Power Applications, vol. 10, no. 7, pp. 593-597, 2016

[26] C. Nordling and J. Österman, Physics Handbook for Science and Engineering, 8th ed. Lund, Sweden: Studentlitteratur, 2006. 